\title{
Family conflict, chaos, and negative life events predict cortisol activity in low-income children
}

\author{
Jenalee R. Doom ${ }^{1}$ (D) | Stephanie H. Cook $^{2}$ | Julie Sturza ${ }^{1}$ | Niko Kaciroti ${ }^{1,3}$ | \\ Ashley N. Gearhardt ${ }^{4}$ | Delia M. Vazquez ${ }^{1,5}$ | Julie C. Lumeng ${ }^{1,5,6}$ | \\ Alison L. Miller ${ }^{1,7}$
}

${ }^{1}$ Center for Human Growth and

Development, University of Michigan, Ann

Arbor, Michigan

2 Departments of Biostatistics and Social and Behavioral Health, New York University

College of Global Public Health, New York,

New York

${ }^{3}$ Department of Biostatistics, University of Michigan School of Public Health, Ann Arbor, Michigan

${ }^{4}$ Department of Psychology, University of

Michigan, Ann Arbor, Michigan

${ }^{5}$ Department of Pediatrics, University of Michigan Medical School, Ann Arbor, Michigan

${ }^{6}$ Department of Nutritional Sciences, University of Michigan School of Public Health, Ann Arbor, Michigan

7 Department of Health Behavior and Health Education, University of Michigan School of Public Health, Ann Arbor, Michigan

\section{Correspondence}

Jenalee Doom, PhD, Center for Human Growth and Development, University of Michigan, 300 N. Ingalls St., Ann Arbor, MI 48109.

Email: jrdoom@umich.edu

Funding information

National Institute of Diabetes and Digestive and Kidney Diseases, Grant numbers: R01DK095695, R21DK090718, RC1DK086376; American Heart Association, Grant number: 10GRNT4460043; Eunice Kennedy Shriver National Institute of Child Health and Human Development, Grant number: F32HD088029

\begin{abstract}
Childhood poverty is hypothesized to increase risk for mental and physical health problems at least in part through dysregulation of the hypothalamic-pituitary-adrenal axis. However, less is known about the specific psychosocial stressors associated with cortisol reactivity and regulation for children living in poverty. The current study investigates negative life events, household chaos, and family conflict in preschool and middle childhood as potential predictors of cortisol regulation in low-income 7-10 year olds $(N=242 ; M$ age $=7.9$ years). Participants were assessed in preschool and participated in a follow-up assessment in middle childhood, during which diurnal free cortisol and free cortisol reactivity to the Trier Social Stress Test for Children (TSST-C) were assessed. Household chaos during preschool predicted a more blunted diurnal cortisol slope in middle childhood. Greater negative life events during preschool and greater concurrent family conflict were associated with increased free cortisol reactivity in middle childhood.
\end{abstract}

\section{KEYWORDS}

chaos, childhood, cortisol, family conflict, negative life events, poverty

\section{1 | INTRODUCTION}

Childhood poverty has been associated with increased rates of mental and physical health problems throughout the lifespan (Repetti, Taylor,
\& Seeman, 2002). One of the hypothesized mediators between childhood poverty and later health problems is through dysregulation of the hypothalamic-pituitary-adrenal (HPA) axis due to stressors associated with living in poverty. The HPA axis is a coordinator of 
physiological systems both under stress and at basal levels, a critical regulator of development, and a mechanism by which stress "gets under the skin" to affect mental and physical health (Gunnar, Doom, \& Esposito, 2015; Gunnar \& Quevedo, 2007). Childhood poverty has consistently been associated with disruptions in the HPA axis (e.g., Evans \& Kim, 2007; Lupien, King, Meaney, \& McEwen, 2001), but these findings have been mixed, with low SES associated with both higher (Evans \& Kim, 2007; Lupien et al., 2001; Vliegenthart et al., 2016) and lower/blunted (Blair, Berry, Mills-Koonce, \& Granger, 2013) basal cortisol levels. Studies of cortisol reactivity to stress in relation to poverty are less common. Blair, Granger, and Razza (2005) have reported that a lower income-to-needs ratio in 4- to 5-year olds was associated with greater cortisol reactivity to a lab assessment. In addition, children in the intervention group of a cash-transfer program in Mexico showed lower salivary cortisol levels at ages 2-6 years than those in the control group (Fernald \& Gunnar, 2009). Findings vary depending on child age at assessment and exposure, perhaps because many studies have not considered different types of stress exposure among low-income children. Childhood poverty encompasses a variety of stress exposures and experiences, including negative life events, family conflict and chaos (Evans, 2004), and researchers must understand which psychosocial stressors are most likely to disrupt different components of stress-mediating systems to inform interventions for children from low-income backgrounds.

\section{2 | SPECIFICITY OF STRESSOR TYPE}

The Allostatic Load Model (ALM) argues that chronic exposure to heightened neuroendocrine and neural responses to stress over time gradually result in wear and tear on a number of physiological systems, including the neural, HPA, and cardiovascular systems, to produce dysfunction in mental and physical domains (McEwen \& Stellar, 1993). Although cumulative risk models like the ALM are extremely useful for understanding stressful experiences more broadly and assessing the overall level of stress experienced (Evans, Li, \& Whipple, 2013), there is of course a loss of specificity when trying to understand how certain types of stressors individually affect the HPA axis. The HPA axis responds to a variety of threats, including physical danger, disruption in social relationships, and threats to the social self (Dickerson \& Kemeny, 2004; Feng et al., 2011). Threats that are unpredictable, uncontrollable, self-evaluative, and severe are typically the most potent for producing measurable stress responses (Dickerson \& Kemeny, 2004). Poverty affects the HPA axis in significant but heterogeneous ways, and it could be that variation in the type and timing of stressors associated with poverty impact cortisol production in different ways. This could be due to differences in the severity and chronicity of the threat, the interpretation of the threat (e.g., whether it threatens the social self), the age of the individual, and the availability of social buffers, among other factors.

Our current understanding of stress-mediating systems suggests that stress responses are usually not highly correlated with one another (Doom \& Gunnar, 2013). For example, HPA responses are not typically highly correlated with measures of autonomic activity, and diurnal cortisol production is not always related to cortisol reactivity to stress (Doom \& Gunnar, 2013). In the HPA system, two types of receptors for cortisol are utilized, with mineralocorticoid receptors mediating basal genomic activity and glucocorticoid receptors mediating genomic activity in response to stress (for review, see Gunnar \& Vazquez, 2006). Different aspects of the environment likely affect different components of cortisol production, as basal cortisol and cortisol reactivity are not always associated with stressors and problem behaviors in the same manner (e.g., Alink et al., 2008; Tomiyama et al., 2012). Little is known about how stressors jointly affect diurnal cortisol and cortisol reactivity in children as few studies measure both basal cortisol levels and cortisol reactivity. In addition, the heterogeneity in stress responses has challenged researchers to contemplate how and why the stress system reacts uniquely to stressors with different characteristics and how these responses change over time (Joëls \& Baram, 2009). In the context of poverty, household chaos, negative life events, and family conflict could all affect the HPA axis in different ways. For example, family conflict is likely interpreted, either consciously or unconsciously, as a stressor that is threatening to both the child and family stability. As a result, children exposed to family conflict might be hypervigilant to social threats, have more negative interpretations and emotional reactions, and display a heightened autonomic and HPA response when confronted with future social threats (Luecken, Roubinov, \& Tanaka, 2013; Miller, Chen, \& Parker, 2011). On the other hand, chaos in the family may be consciously or unconsciously interpreted as an unpredictable and uncontrollable stressor, but not necessarily a severe stressor that would threaten the child or the family. As a result, household chaos might not interfere with cortisol reactivity to social stressors, but it may disrupt diurnal cortisol regulation if the child must respond to multiple mild stressors throughout the day or if chaos disrupts daily routines that typically help regulate children's stress biology (Gunnar \& Vazquez, 2001).

Many studies have examined social-contextual and relational stressors separately (described below), but few have tested the unique associations of multiple stressors in the same population. Importantly, no study to our knowledge has examined the impact of multiple stressors longitudinally on cortisol reactivity and diurnal cortisol regulation in children from low-SES backgrounds. Filling this gap in the literature will help us to understand inconsistent findings in the poverty and cortisol literature and spur future research on the role of specific effects of stressors in development. Potential stressors that affect HPA regulation in children from low-SES backgrounds are described below.

\section{1 | Negative life events}

Children in low-income households have a high likelihood of experiencing negative life events (Evans, 2004). Exposure to such events can trigger stress responses, and over time, this may shape differences in diurnal cortisol regulation and cortisol reactivity from repeatedly responding to acute highly stressful events. Negative life events are different from more chronic stressors in that they are discrete events that may negatively impact the child and family but do not necessarily lead to chronic dysfunction. Lifetime trauma exposure has been associated with increased basal hair cortisol in children (Simmons et al., 2016). In a population study of adolescents, adversities from 0 to 5 years were not 
associated with cortisol reactivity but adversities from age 6-11 years were associated with higher cortisol reactivity, indicating the importance of developmental timing (Bosch et al., 2012). Although there has been increased interest in effects of negative life events on health, there is a significant gap in the literature examining how negative life events impact both basal cortisol and cortisol reactivity in middle childhood.

\section{2 | Chaos}

In addition to exposure to acute negative events, a generally chaotic home environment may affect cortisol regulation by creating a chronically unpredictable environment that requires constant vigilance (Miller et al., 2011), hindering normative development of stress regulatory systems in early childhood. Household chaos differs from negative life events in that it is less imminently threatening to the child and may instead be perceived as a chronic state of unpredictability and uncontrollability, producing frequent mild stressors rather than less frequent severe stressors. Chen, Cohen, and Miller (2010) reported that children and adolescents from low SES homes showed greater increases in diurnal cortisol output across 2 years than children from high SES homes, which was partially mediated by family chaos. Likewise, Sturge-Apple, Davies, Cicchetti, Hentges, and Coe (2017) reported higher family instability in the context of poverty is associated with greater morning basal cortisol levels in 2-year-old children. However, in the preschool wave of the current cohort, children from homes with more compared to less socioemotional chaos demonstrated lower morning cortisol (Lumeng et al., 2014). Most studies in children have focused on the impact of chaos on basal cortisol, and as a result, there is a significant gap in the literature on how chaos in middle childhood is associated with cortisol reactivity.

\section{3 | Family conflict}

Family conflict may contribute to cortisol dysregulation by decreasing the effectiveness of parents to help children regulate stress and of the child to form stress-buffering social relationships (Miller et al., 2011). Family conflict is different from negative life events and chaos because of its social nature. This social stressor could lead children to consciously or unconsciously interpret conflict as being threatening to the family's safety and stability. In kindergarteners, greater interparental conflict was associated with blunted cortisol reactivity to simulated parental conflict (Davies, Sturge-Apple, Cicchetti, \& Cummings, 2007). Adolescents with high family aggression levels demonstrated blunted cortisol reactivity to more versus less conflictual discussions with parents (Saxbe, Margolin, Spies Shapiro, \& Baucom, 2012). In 3-5 year olds, greater child conflict in the home was associated with lower awakening cortisol and a flatter diurnal cortisol slope across the day (Slatcher \& Robles, 2012). One study reported that children in families with higher marital discord had higher wake-up and average cortisol levels across the day (Pendry \& Adam, 2007). Children in families with high marital discord also had a less pronounced decrease in cortisol levels across the day and higher bedtime cortisol compared to children in families with low marital discord, and this effect was more pronounced for kindergarten-aged children than for adolescents (Pendry \& Adam, 2007).

These three types of stressors (negative life events, chaos, and family conflict) may affect basal cortisol and cortisol reactivity through different pathways, but no study to our knowledge has examined these pathways with respect to both basal cortisol and cortisol reactivity in middle childhood. In addition, the developmental timing of these stressors in relation to cortisol production in middle childhood is unclear.

\section{3 | DEVELOPMENTAL TIMING AND TIMING OF STRESSORS}

We have a myriad of evidence that chronic HPA activation produces heightened cortisol levels close in time to the stressor, but downregulation of the HPA axis in response to chronically high cortisol levels produces normal to low cortisol levels, even if higher levels of the axis (e.g., hypothalamic corticotropin-releasing hormone; $\mathrm{CRH}$ ) and limbic regions are still hyper-responding (Fries, Hesse, Hellhammer, \& Hellhammer, 2005). Thus, the timing of stress is important to understanding current cortisol production, as recent stressors may have a profile of hyperactivation while more distal stressors may have a profile of hypoactivation (Doom \& Gunnar, 2013; Miller, Chen, \& Zhou, 2007). Compounding this issue of timing is the realization that the developing brain and stress-mediating systems may respond to stressors differently in childhood than in adolescence or beyond. The developmental timing of early risk factors may differentially affect current and future cortisol regulation, and may be particularly important in early childhood when stress regulation systems are established (Gunnar \& Quevedo, 2007; Lupien, McEwen, Gunnar, \& Heim, 2009). Another important developmental concern is that stressors may change over time, and it may be that an increasing or decreasing amount of stress may better predict cortisol production than stress at a particular time point.

\section{CURRENT STUDY}

The goal of this investigation is to understand what components of stress exposure (e.g., negative life events, chaos, and family conflict) affect diurnal cortisol and cortisol reactivity longitudinally in lowincome children. Although these three aspects of the environment have been examined in relation to basal HPA functioning (usually diurnal cortisol slope or hair cortisol), to our knowledge this is the first study to examine these factors together in relation to both diurnal cortisol and cortisol reactivity within a sample of low-income children in middle childhood. This study will model these parallel stress processes that could lead to individual differences in cortisol production in a group of low-income children. It is hypothesized that more negative life events, chaos, and family conflict in preschool will be associated with lower morning cortisol and a blunted diurnal cortisol slope in middle childhood. As there are few data examining cortisol reactivity in low-income children in middle childhood, we tentatively predict that greater current negative life events will be 
associated with heightened cortisol reactivity, while greater preschool family conflict will be associated with blunted cortisol reactivity.

\section{5 | METHODS}

\section{1 | Participants}

A total of 242 children who participated in the middle childhood follow-up of a longitudinal study are included in analyses (see Table 1 for participant demographics). Children were first recruited in preschool through their participation in Head Start, a federally funded education and health program for low-income children in the United States. Children and their primary caregiver (92\% mothers) were recruited through a form sent home with the child from Head Start. Parents who returned the demographic form and contact information were compensated with $\$ 10$. Parents were then contacted to determine study eligibility and interest in participation. Exclusion criteria included: child or parent was unable to complete informed consent in English; primary caregiving parent with $\geq 4$-year college degree in order to target a low-income sample; child in foster care; child had food allergies, significant medical problems, or perinatal complications; gestational age $<35$ weeks. Children were included in

TABLE 1 Participant characteristics

\begin{tabular}{|c|c|c|c|}
\hline & M & SD & $\%$ \\
\hline T1 age first session (months) & 51.26 & 6.11 & \\
\hline T1 age second session (months) & 58.73 & 8.28 & \\
\hline Female & & & 51.2 \\
\hline T2 pubertal status (females) & 1.33 & 0.51 & \\
\hline \multicolumn{4}{|l|}{ Child race/ethnicity } \\
\hline Non-Hispanic white & & & 53.7 \\
\hline African American & & & 16.9 \\
\hline Hispanic/Latino & & & 10.3 \\
\hline \multicolumn{4}{|l|}{ Parent education } \\
\hline Did not graduate high school & & & 16.4 \\
\hline High school degree or GED & & & 29.7 \\
\hline Some college courses & & & 40.5 \\
\hline 2-year college degree & & & 13.4 \\
\hline T1 income-to-needs ratio & 0.87 & 0.69 & \\
\hline T2 income-to-needs ratio & 1.10 & 0.76 & \\
\hline T1 negative life events (number) & 4.03 & 3.30 & \\
\hline T1 negative life events (sum of how bothered across events) & 5.09 & 6.13 & \\
\hline T2 family conflict & 1.72 & 0.65 & \\
\hline T2 sleep disturbance & 45.35 & 7.09 & \\
\hline T2 baseline perceived stress & 1.36 & 0.78 & \\
\hline T2 perceived stress during task & 3.57 & 1.32 & \\
\hline T2 perceived stress after task & 1.10 & 0.37 & \\
\hline
\end{tabular}

Means, standard deviations, and percentages of participants' demographic information and key variables for individuals who were included in the analyses. T1, preschool assessment; T2, middle childhood assessment. GED, General Educational Development Test (high school equivalency test in the United States). 
the current analysis if they had valid cortisol data for the reactivity protocol or the diurnal saliva collection. We excluded participants who either reported inhaler use in the past $24 \mathrm{hr}$ or the daily use of a corticosteroid ( $N=10$; total $N$ does not include these participants), which could interfere with cortisol values. Children included in the current study did not differ from those who participated in earlier waves or those who did not provide cortisol in middle childhood as a function of the following T1 measures: sex, income-to-needs ratio, primary caregiving parent education, race/ethnicity, or BMIz, all ps $>.05$. Children with valid cortisol data in middle childhood were slightly older ( $M=51.3$ months) than those who did not have valid cortisol data or did not participate in the middle childhood assessment ( $M=49.7$ months), $p<0.05$. This study was approved by the university's institutional review board.

\section{$5.2 \mid$ Procedure}

Children and parents participated in sessions at three time points: two in preschool (1st session age 35-62 months, $M=50.6$ months, $\mathrm{SD}=6.4$, median $=51.7, N=380 ; 2 \mathrm{nd}$ session age 38-85 months, $M=58.7$ months, $S D=8.5$, median $=59.2 ; N=330$ ) and one in middle childhood (age 84-122 months, $M=95.0$ months, $S D=8.6$, median $=93.7 ; N=275$ ). The preschool sessions ranged from 0 to 26.0 months apart $(M=7.9$ months, $S D=5.6$, median $=6.4)$. The time between the first preschool and the middle childhood sessions ranged from 28.1 to 65.4 months apart ( $M=44.4$ months, $S D=7.9$, median $=43.6$; see Table 1 for descriptive information for participants in the current analyses). At the first preschool assessment, questionnaires were completed by the parent to assess demographics, income, and chaos. At the second preschool assessment, parents completed questionnaires on current family conflict and negative life events in the past year. At the middle childhood assessment, parents completed questionnaires on demographics, income, chaos, family conflict, and negative life events in the past year. In addition, diurnal free cortisol and free cortisol in response to stress were assessed.

The parent provided written consent and children provided ageappropriate assent to participate. Families were compensated for their time. Research assistants (bachelor-level) administered questionnaires to parents and collected all saliva samples from children during the stress protocol. Parents collected saliva at home for the diurnal free cortisol assessment.

\section{3 | Saliva collection}

\subsection{1 | Diurnal salivary cortisol}

Parents were sent home with a saliva collection kit to collect their child's saliva three times per day for 3 days. The morning sample was to be around 8:00 am before breakfast and school, within $30 \mathrm{~min}$ of waking (78\% of samples were collected during the first $30 \mathrm{~min}$, and $95 \%$ were collected within the first hour of waking). The after-school sample was to be around $4: 00$ pm before a snack or dinner $(67 \%$ of samples were collected between 3-5:00 pm). The bedtime sample was to be collected around 8-9:00 pm (70\% of samples were collected between 7:30-9:30 pm). We utilized MEMS caps to check parent report of saliva time against the cap-recorded time and found that parents were $92 \%$ accurate at recording log times within $15 \mathrm{~min}$ of the actual time and $94 \%$ accurate at recording times within 30 min of the actual time. Parents were instructed not to let the child eat for 30-45 min before collecting the sample, to space samples at least $3 \mathrm{hr}$ apart, and not to collect saliva if the child was sick. The parent had the child rinse his or her mouth and then chewed on dental cotton for 1-2 min. The parent was instructed to place the sample in a colorcoded tube, mark the time of sample/last meal, and any medications or sickness (in case the parent collected saliva while the child was sick), and place the tube in the freezer until a research assistant could pick up the samples, usually within a week of collection. A research assistant called or texted the parent at the scheduled sample time to answer questions and confirm that the sample was obtained.

\subsection{2 | Salivary cortisol reactivity}

All stress reactivity sessions were conducted in the afternoon (approximately 3-7:00 pm), typically within a week of the diurna saliva collection. Saliva sample \#1 was collected by the research assistant $45 \mathrm{~min}$ after a snack that was followed by calming free play by chewing on a piece of dental cotton for 1-2 min. The child then transitioned to the stress task. The stress reactivity protocol consisted of approximately $10 \mathrm{~min}$ of academic testing with a strict teacher and 10 min of the modified Trier Social Stress Test for Children (TSST-C; Buske-Kirschbaum et al., 1997). The strict teacher was a female research assistant instructed not to give the child any positive feedback and to always use a neutral, but not harsh, tone. The research assistant introducing the teacher told the child that the teacher was very strict, and acted nervous around the teacher. When the teacher entered the room, she made several very slight adjustments to the room setup to show she was picky about rules. The child was told that if he/she did better than the other children tested, he/she would earn a prize at the end.

The strict teacher administered the forward and backward digit span tasks and the oral word fluency task from the Wechsler Individual Achievement Test-Third Edition (WIAT-III, 2009). Then, an adapted TSST-C story book task was administered by the teacher. The child was instructed to tell a detailed story about pictures in the book for $30 \mathrm{~s}$ each (10 total pictures) and was given an example of how to do so. The teacher beeped the timer at the beginning and end of the $30 \mathrm{~s}$. After the 1st and 5th pictures, the teacher said to stop and told the child to say more about the next pictures and speak into the microphone. On all other pages, the teacher would move onto the next picture after $30 \mathrm{~s}$. If the child stopped talking, the teacher would use the prompt, "Keep going," in a neutral voice for a maximum of three times per task.

After 10 pictures, the teacher moved on to the math problem solving portion, reading aloud grade-appropriate questions from the WIAT-III. The child was provided with a paper and pencil. The task was discontinued after four consecutive incorrect answers, and the child 
was not given any feedback in order to keep the task uncertain. If the child was unresponsive for $30 \mathrm{~s}$, he/she was prompted to answer. If the child asked for help, the teacher would respond that she could not help. After the task was discontinued, the teacher told the child she was going to score the answers to see if the child had won the prize and left the room.

Two minutes after the teacher left, the research assistant reentered and asked the child to self-report his or her subjective distress. Saliva sample \#2 was collected immediately after the distress assessment (20-25 min after the start of the stress task). The child was debriefed by the research assistant and the teacher and told that the teacher was practicing how to be strict, and the teacher asked the child whether she had done a good job. The child was given a prize and then played calm games with the research assistant while remaining seated. Sample \#3 was collected about 15 min after the child was debriefed. Sample \#4 was collected about 35-40 min after the debriefing. Saliva was sampled at these multiple time points following the stress-elicitation challenge tasks to capture individual differences in response time for free cortisol reactivity and recovery (Dickerson \& Kemeny, 2004; Lopez-Duran, Hajal, Olson, Felt, \& Vazquez, 2009).

\section{4 | Cortisol assay}

Following collection, saliva samples were thawed completely, vortexed, centrifuged at 3,000 rpm for $15 \mathrm{~min}$, separated from debris, and placed in Thermo Scientific Matrix Racks at $-80^{\circ} \mathrm{C}$ until assayed. Saliva samples were submitted to the University of Michigan Center of Chemical Genomics (CCG) to perform assays. Assays were conducted by the same technician using the same equipment. On the day of the assay, the sample was submitted to the steps for cortisol detection following manufacturer's instructions. Cortisol was assayed using an Expanded Range High Sensitivity Salivary Cortisol Enzyme Immunoassay Kit (Catalog No. 1-3002, 96-Well Kit, Salimetrics LLC, State College, PA) with a detection limit of $0.007 \mu \mathrm{g} / \mathrm{dl}$. The average interassay coefficient of variation (CV) was $4.0 \%$ and the intra-assay CVs ranged from $0.8 \%$ to $6.1 \%$. We report free cortisol in $\mu \mathrm{g} / \mathrm{dl}$.

\section{5 | Questionnaires}

\subsection{1 | Subjective distress}

Children were asked to rate their subjective feelings of distress at three time points to ensure the task was significantly challenging: right before the stress task, during the task (asked 2 min after the task ended), and at the time of the last saliva sample. Responses ranged from 1 (very calm or relaxed) to 5 (very nervous, scared, or stressed out). Analyses indicate that participants felt significantly more stressed during the task than before or after, $p s<0.001$ (means presented in Table 1).

\subsection{2 | Medications}

Parents were asked at the middle childhood assessment whether their child regularly takes any medications for use in analyses. We scored medication use from a 0-2 scale with guidance on medications with possible effects on cortisol (Granger, Hibel, Fortunato, \& Kapelewski, 2009). A value of 0 was assigned for children with no medications or medications with no effect on cortisol. A value of 1 was assigned for children with medications with a possible effect on cortisol. A value of 2 was assigned for children with medications with a likely effect on cortisol, including those with occasional but not daily use.

\subsection{3 | Puberty}

To control for the possible influence of pubertal development on cortisol levels, parents estimated their child's pubertal development based on a visual rating scale for Tanner staging (Morris \& Udry, 1980) at the middle childhood session. Short descriptions of physical changes at each stage were displayed below the photos. Parents of females completed the ratings for breast and pubic hair development, and parents of males completed the rating for genital and pubic hair development. Parents rated their child's development from 1 (not started developing) to 5 (fully developed). For males, the genital and pubic hair development score was used, and for females, the average of the breast and pubic hair development scores was used.

\section{$5.5 .4 \mid \mathrm{BMI}$}

Trained research staff measured child weight and height at the middle childhood assessment. Body mass index (BMI) in middle childhood was calculated as kilograms over meters squared, and BMI z-score (BMIz) was calculated based on the reference growth charts for age and sex from the US Centers for Disease Control and Prevention (Kuczmarski et al., 2002).

\subsection{5 | Sleep disturbance}

To control for potential associations of sleep disturbance with cortisol outcomes (Kumari et al., 2009), the Children's Sleep Habits Questionnaire (CSHQ; Owens, Spirito, \& McGuinn, 2000) was completed by parents in middle childhood. Subscales included bedtime resistance, sleep onset delay, sleep duration, sleep anxiety, night wakings, parasomnias, sleep disordered breathing, and daytime sleepiness. Parents indicated how often an event happened in the past week. If the past week was atypical, they were instructed to think about the most recent typical week. Rarely (0-1 times) was coded as 1 , sometimes (2-4 times) as 2 , and usually (5-7 times) as 3 . The total sleep disturbance score is the sum of 33 items from the 8 subscales, and this total score was used in analyses. In this sample, the score ranged from 34 to 79. This scale has been used extensively to identify sleep problems in school-aged children (Owens et al., 2000).

\subsection{6 | Negative life events}

The 30-item Life Events Checklist (LEC) was completed by the parent at both the preschool (second session) and middle childhood assessments to report what negative events happened to the family in the 
past year (Kilmer, Cowen, Wyman, Work, \& Magnus, 1998; Work, Cowen, Parker, \& Wyman, 1990). Example items include family member with a serious illness, family member arrested or in jail, death of family, parents separated or divorced, family member with an alcohol/drug problem, and neighborhood violence. Participants respond with yes or no for each item, and if they responded with yes, they were then asked to report how much the child was bothered or upset by this event. Responses included not at all bothered (receiving a score of 0 ), a little bit, somewhat, and a lot (score of 3 ). The final score for the impact of stressful life events was calculated by summing the responses for the "bothered" questions for all events that were endorsed for a potential range of 0-90. Those who did not endorse any events received a 0 on this scale. We chose to use how "bothered" children were by the event instead of the total number as it is arguably a more person-centered approach for capturing the child's experience (Jenness \& McLaughlin, 2015). In this sample, the scale ranged from 0 to 30 at the preschool assessment and 0-36 at the middle childhood assessment.

\subsection{7 | Chaos}

The parent completed the 15-item Chaos, Hubbub, and Order Scale (CHAOS; Matheny, Wachs, Ludwig, \& Phillips, 1995) at both the preschool (first session) and middle childhood sessions to assess general chaos in the home. This scale assessed a number of aspects of the home, including level of commotion, the home is a "zoo," being able to talk without being interrupted, often a fuss going on, unable to hear yourself think, getting drawn into others' arguments, able to relax, always seem to be rushed, able to stay on top of things, having a regular routine, and calm atmosphere. This scale has demonstrated good internal consistency, test-retest reliability, and external validity (Matheny et al., 1995). If an item indicating greater chaos was endorsed as true, it was assigned a value of 1 , and it was assigned a value of 0 for false. The number of items endorsed was summed for the final score (range: 0-15). If items were missing, the average score across the 15 variables was used. A higher score indicated a higher level of chaos in the home.

\subsection{8 | Family conflict}

Conflict in the family was assessed at both the preschool (second session) and middle childhood assessments using the 12-item conflict scale of the Self-Report Family Inventory (Beavers, Hampson, \& Hulgus, 1991). Items of the conflict scale measure overt conflict, such as openly fighting, blaming, arguing, negative tone, and unresolved conflicts. Responses ranged from 1 = fits our family well to $5=$ does not fit our family at all. Responses were reversed for appropriate items and then averaged across the 12 items such that a higher score indicated greater family conflict. Scores could range from 1 to 5 , but in this sample ranged from 1 to 4.33 at the preschool session and from 1 to 4.25 at the middle childhood session. The overall inventory has demonstrated good reliability and validity with other measures of family functioning (e.g., Hampson, Hulgus, \& Beavers, 1991).

\subsection{9 | Income-to-needs}

The immediate family's income-to-needs ratio was used at the preschool (1st session) and middle childhood assessments to test whether associations were specific to negative life events, chaos, and family conflict rather than to low income. Annual pre-tax income from all sources was reported by the parent, who was asked to report their income or to choose a category that best represented their family. There were 18 categories that participants were sorted into based on their response, ranging from less than $\$ 5,000$ to more than $\$ 200,000$. This dollar amount was then divided by the poverty threshold for a family of the same size to arrive at the income-to-needs ratio. The mean of 0.87 for this sample during preschool indicated that families were generally living in poverty.

\subsubsection{0 | Demographics}

The parent who completed the questionnaires also reported his or her highest level of schooling at the first preschool assessment as (i) did not finish high school; (ii) high school diploma or US high school equivalency test (General Educational Development test; GED); (iii) some college courses; or (iv) 2-year college degree. The child's race and ethnicity were reported by the parent and coded as non-Hispanic white $=0$, Hispanic and/or non-white $=1$ for analysis. Child sex (male vs. female) was reported by the parent and included in all models as sex differences in HPA functioning in low-income children have been reported even before puberty (e.g., Doom, Cicchetti, Rogosch, \& Dackis, 2013).

\section{6 | Data analytic plan}

\subsection{1 | Diurnal free cortisol data}

As recommended in the field (e.g., Gunnar \& White, 2001; Massey et al., 2016) and in preparation for analyses, we removed any saliva sample for which the free cortisol value deviated more than three standard deviations from the calculated mean of a specific time point. Following this procedure, 43 of the 2,162 samples assayed (2.0\%) were excluded. Children who had at least five saliva samples across at least 2 days were included in the analysis in order to create diurnal curves that accurately represented the child's diurnal cortisol pattern on more than 1 day (mean number of data points per child $=8.4, S D=1.1 ; 6$ children were excluded for this reason).

As in prior work, we used hierarchical linear modeling (HLM) to capture diurnal free cortisol curves for each participant by generating random parameters with the restricted maximum likelihood method (REML; Lumeng et al., 2014; Miller et al., 2017). Provided that trajectories have a known parametric form, the HLM approach is a powerful technique for estimating individual trajectories (Hruschka, Kohrt, \& Worthman, 2005). This approach can directly account for differential measurement of cortisol if sampling times are not uniform by using the parametric function of the known diurnal pattern. HLM is a robust estimation method, even with missing data. Using minutes as 
the independent variable (derived from parent-reported time since awakening) and log-transformed cortisol as the outcome, the diurnal pattern obtained is linear on time in a log-scale (for time $\geq 60 \mathrm{~min}$ ) and is captured by the intercept and slope of the derived line. Thus, the random intercept generated by the HLM is an estimate of the expected 60 min post-awakening free cortisol level for an individual, and the random slope generated by the HLM is the expected rate of cortisol decay from 60 min post-awakening onward. Preliminary analyses on the data obtained indicated that cortisol values were sensitive to whether or not the child ate before the sample. As a result, we controlled for whether the child ate before each of the samples in the HLM model. Recall that each child provided samples for 3 days. Each free cortisol measurement on each day was included in the model, along with the corresponding time since awakening for that particular day and the sample time. Random effect parameters estimated each child's expected cortisol pattern over the 3 days that were sampled, giving a single predicted cortisol intercept and slope for each child while taking into account data from all 3 days. The random intercept and slope were used as individual-level dependent variables for the analyses.

\subsection{2 | Cortisol reactivity data}

Again, in preparation for analyses, we removed any saliva sample for which the cortisol value deviated more than three standard deviations from the calculated mean of a specific time point (e.g., Massey et al., 2016). Following this procedure, 18 of the 899 samples assayed (2.0\%) were excluded. We assessed the cortisol response to stress by calculating the area under the curve (AUCi). The cortisol AUCi was calculated using the trapezoidal rule and reflected the child's total free cortisol output increase from the first sample (prior to starting the stressor) to the fourth sample ( $40 \mathrm{~min}$ after conclusion of challenging tasks). AUCi is typically used in this manner as an indicator of overall stress response (Pruessner,
Kirschbaum, Meinlschmid, \& Hellhammer, 2003). For follow-up analyses, AUCi was separately calculated for reactivity (Times 1 to 2 ) and recovery (Times 2 to 4 ).

\subsection{3 | Diurnal cortisol and cortisol reactivity models}

The final regression analyses were completed in SPSS version 24. A correlation table of model variables can be found in Table 2 . Three regression models were calculated with cortisol intercept (estimated 60 min post-awakening), diurnal cortisol slope, and cortisol $\mathrm{AUCi}$ (area under the curve with respect to increase) in response to stress as dependent variables. The final models were created by first including preschool and middle childhood negative life events, chaos, family conflict as independent variables. Sex ( male $=1$, female $=2$ ) and race/ethnicity (non-Hispanic white $=0$, Hispanic and/or nonwhite $=1$ ) were added as covariates in addition to the following covariates assessed at middle childhood: age at assessment, current medications, pubertal development, sleep disturbance, BMI z-score, and income-to-needs ratio (in both preschool and middle childhood). The random cortisol intercept was considered as a covariate in the diurnal slope analysis, and time since awakening on the day of the reactivity protocol was considered as a covariate for the reactivity analysis. Independent variables and covariates were removed from the analysis for parsimony if they were not independent predictors of either diurnal cortisol intercept, slope, or cortisol AUCi, $p<.10$. Independent variables and covariates were also tested to ensure that exclusion did not change the direction or significance of the model, $p<.10$. Thus, the final models for intercept, slope, and AUCi had identical predictors (except for cortisol intercept for the slope model and time since waking for the AUCi model, which were included if they were significant predictors of the outcome or their exclusion did not change the model). Results are displayed as T1 (Time 1: preschool assessments) or T2 (Time 2: middle childhood assessment) for each variable.

TABLE 2 Correlation table

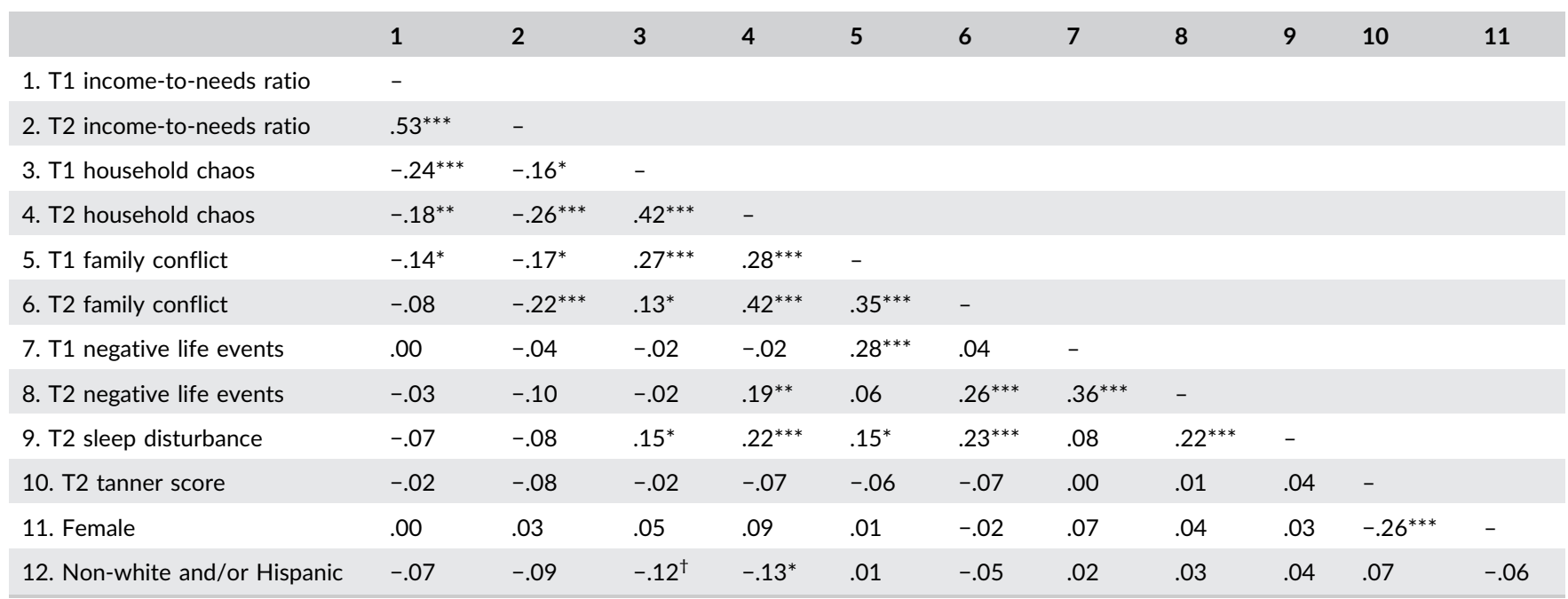

Pearson correlation coefficients with statistical significance indicated by ${ }^{\dagger} p \leq 0.10,{ }^{*} p \leq 0.05,{ }^{* *} p \leq 0.01,{ }^{* * *} p \leq 0.001$. T1, preschool assessment; T2, middle childhood assessment. 
In order to test whether change in the stressors (i.e., increasing chaos) rather than the level of the stressor at either time point was more associated with cortisol measures, change scores were added to the final model to test whether they predicted the outcomes. Each of the three stressors (life events, chaos, and family conflict) at both time points were $z$-scored within the sample, and the T1 score was subtracted from the T2 score to create the change score for that particular stressor. Nonsignificant change variables were removed from the final model, $p<.10$.

Follow-up analyses were conducted to test whether predictors of cortisol AUCi are more associated with cortisol reactivity (AUCi from Times 1 to 2) or recovery (AUCi from Times 2 to 4). The model predictors that were derived from the models of $\mathrm{AUCi}$, diurnal cortisol intercept, and diurnal cortisol slope were used to predict reactivity and recovery.

\section{6 | RESULTS}

\section{$6.1 \mid$ Initial analyses}

The AUCi for reactivity (Times 1 to 2 ) was significantly larger than 0 , mean difference $=46.62, t(194)=4.55, p<0.001$, indicating an overal cortisol response to the stressor. The morning free cortisol intercept and diurnal slope were significantly correlated, $r=.49, p<.001$. Free cortisol reactivity to stress was not correlated with the morning free cortisol intercept, $r=.00, p=.96$, or the diurnal slope, $r=.05, p=.49$.

\section{$6.2 \mid$ Morning cortisol intercept}

The final model predicting the T2 cortisol intercept $(60 \mathrm{~min}$ postawakening) included T2 pubertal status, T2 age, sex, race/ethnicity, T2 sleep disturbance, T2 BMIz, T1 income-to-needs ratio, T1 negative life events, T1 chaos, T1 family conflict, and T2 family conflict (see Table 3). The overall model significantly predicted the T2 cortisol intercept, $F(11,172)=2.22, p=.02$. Females showed higher T2 cortisol intercepts than males, $t(172)=2.65, p<.01$, those with higher $\mathrm{BMIz}$ showed lower T2 cortisol intercepts, $t(172)=-3.02, p<.01$, and older participants demonstrated lower T2 cortisol intercepts, $t(172)=-2.46$, $p=.02$. Higher T2 pubertal status, $t(172)=1.67, p=.098$, and greater T2 family conflict, $t(172)=1.86, p=0.07$, predicted a greater cortisol intercept at the level of a trend. No other variables in the model were significant predictors, $p s>.10$. None of the change scores predicted the T2 cortisol intercept, $p s>.10$.

\section{3 | Diurnal cortisol slope}

The final model predicting T2 diurnal cortisol slope consisted of the T2 cortisol intercept, T2 pubertal status, T2 age, sex, race/ethnicity, T2 sleep disturbance, T2 BMIz, T1 income-to-needs ratio, T1 negative life events, T1 chaos, T1 family conflict, and T2 family conflict (see Table 4). The overall model significantly predicted the T2 diurnal cortisol slope, $F(12,171)=7.29, p<.001$. Greater T1 chaos was associated with a more blunted T2 cortisol slope, $t(171)=3.03, p<.01$. Being female and identifying as non-Hispanic white were indepen-
TABLE 3 Cortisol intercept analysis

\begin{tabular}{|c|c|c|c|c|}
\hline & $\begin{array}{l}\text { Unstandardized } \\
\text { B }\end{array}$ & $\begin{array}{l}\text { Unstandardized } \\
\text { SE }\end{array}$ & $\begin{array}{l}\text { Standardized } \\
\beta\end{array}$ & $T$-score \\
\hline $\begin{array}{l}\text { T2 pubertal } \\
\text { status }\end{array}$ & 0.22 & 0.13 & 0.13 & $1.67^{\dagger}$ \\
\hline T2 age & -0.02 & 0.01 & -0.18 & $-2.46^{*}$ \\
\hline Female & 0.39 & 0.15 & 0.20 & $2.65^{* *}$ \\
\hline $\begin{array}{l}\text { Non-white } \\
\text { and/or } \\
\text { Hispanic }\end{array}$ & 0.19 & 0.15 & 0.10 & 1.30 \\
\hline $\begin{array}{l}\text { T2 BMI } \\
z \text {-score }\end{array}$ & -0.23 & .08 & -0.23 & $-3.02^{* *}$ \\
\hline $\begin{array}{l}\text { T2 sleep } \\
\text { disturbance }\end{array}$ & 0.00 & .01 & -0.01 & -0.13 \\
\hline $\begin{array}{l}\text { T1 income- } \\
\text { to-needs }\end{array}$ & 0.13 & 0.11 & 0.09 & 1.22 \\
\hline $\begin{array}{l}\text { T1 negative } \\
\text { life } \\
\text { events }\end{array}$ & 0.01 & .01 & 0.05 & 0.58 \\
\hline $\begin{array}{l}\text { T1 } \\
\text { household } \\
\text { chaos }\end{array}$ & 0.00 & .03 & -0.01 & -0.14 \\
\hline $\begin{array}{l}\text { T1 family } \\
\text { conflict }\end{array}$ & -0.14 & 0.11 & -0.10 & -1.27 \\
\hline $\begin{array}{r}\text { T2 family } \\
\text { conflict }\end{array}$ & 0.23 & 0.12 & 0.15 & $1.86^{\dagger}$ \\
\hline
\end{tabular}

Statistical significance indicated by ${ }^{\dagger} p<0.10,{ }^{*} p<0.05,{ }^{* *} p<0.01$, ${ }^{* * *} p<0.001$. T1, preschool assessment; T2, middle childhood assessment.

TABLE 4 Diurnal cortisol slope analysis

\begin{tabular}{|c|c|c|c|c|}
\hline & $\begin{array}{l}\text { Unstandardized } \\
\text { B }\end{array}$ & $\begin{array}{l}\text { Unstandardized } \\
\text { SE }\end{array}$ & $\begin{array}{l}\text { Standardized } \\
\beta\end{array}$ & $T$-score \\
\hline $\begin{array}{l}\text { Cortisol } \\
\text { intercept }\end{array}$ & 0.48 & 0.06 & 0.50 & $7.53^{* * *}$ \\
\hline $\begin{array}{l}\text { T2 pubertal } \\
\text { status }\end{array}$ & -0.21 & 0.11 & -0.12 & $-1.81^{\dagger}$ \\
\hline T2 age & -0.01 & 0.01 & -0.09 & -1.34 \\
\hline Female & -0.36 & 0.13 & -0.19 & $-2.82^{* *}$ \\
\hline $\begin{array}{l}\text { Non-white } \\
\text { and/or } \\
\text { Hispanic }\end{array}$ & 0.27 & 0.12 & 0.14 & $2.15^{*}$ \\
\hline $\begin{array}{l}\text { T2 BMI } \\
\text { z-score }\end{array}$ & -0.01 & 0.07 & -0.01 & -0.16 \\
\hline $\begin{array}{l}\text { T2 sleep } \\
\text { disturbance }\end{array}$ & 0.00 & 0.01 & 0.00 & 0.00 \\
\hline $\begin{array}{l}\text { T1 income- } \\
\text { to-needs }\end{array}$ & 0.05 & 0.09 & 0.03 & 0.51 \\
\hline $\begin{array}{l}\text { T1 negative } \\
\text { life } \\
\text { events }\end{array}$ & 0.00 & 0.01 & 0.01 & 0.19 \\
\hline $\begin{array}{l}\text { T1 } \\
\text { household } \\
\text { chaos }\end{array}$ & 0.07 & 0.02 & 0.20 & $3.03^{* *}$ \\
\hline $\begin{array}{l}\text { T1 family } \\
\text { conflict }\end{array}$ & -0.06 & 0.10 & -0.04 & -0.61 \\
\hline $\begin{array}{r}\text { T2 family } \\
\text { conflict }\end{array}$ & -0.03 & 0.11 & -0.02 & -0.30 \\
\hline
\end{tabular}

Statistical significance indicated by ${ }^{\dagger} p<0.10,{ }^{*} p<0.05,{ }^{* *} p<0.01$, ${ }^{* * *} p<0.001$. T1, preschool assessment; T2, middle childhood assessment. 
dently associated with a steeper T2 diurnal cortisol slope, $p s<.05$. Being more advanced in puberty was associated with a steeper cortisol slope at the level of a trend, $t(171)=-1.81, p=.07$. Neither T1 nor T2 negative life events or family conflict, nor T2 chaos predicted the T2 cortisol slope, $p s>.10$. None of the change scores predicted the T2 diurnal cortisol slope, $p s>.10$ (Figure 1).

\section{4 | Cortisol AUCi}

The final model predicting T2 cortisol reactivity included T2 pubertal status, T2 age, sex, race/ethnicity, T2 sleep disturbance, T2 BMIz, T1 income-to-needs ratio, T1 negative life events, T1 chaos, T1 family conflict, and T2 family conflict (see Table 5). The overall model significantly predicted T2 cortisol AUCi, $F(11,140)=2.32, p=.01$. Greater T2 family conflict, $t(140)=2.75, p<0.01$, and greater negative life events at $\mathrm{T} 1, t(140)=2.17, p=.03$, were associated with an increased T2 cortisol AUCi. Greater T1 family conflict was associated with lower T2 cortisol AUCi at the level of a trend, $t(140)=-1.81$, $p=.07$. Greater T2 sleep disturbance predicted lower T2 cortisol AUCi, $t(140)=-3.87, p<.001$. No other variables were associated with T2 cortisol AUCi, $p \mathrm{~s}>.10$. The change scores did not predict T2 cortisol $A \cup C i, p s>.10$ (Figure 2).

\section{5 | Follow-up analyses: Cortisol reactivity versus recovery}

The final two models with T2 cortisol reactivity AUCi and cortisol recovery $\mathrm{AUCi}$ as outcomes had the following variables as predictors: T2 pubertal status, T2 age, sex, race/ethnicity, T2 sleep disturbance, T2 BMIz, T1 income-to-needs ratio, T1 negative life events, T1 chaos, T1 family conflict, and T2 family conflict. The overall model significantly predicted cortisol reactivity, $F(11,140)=2.14, p=0.02$.

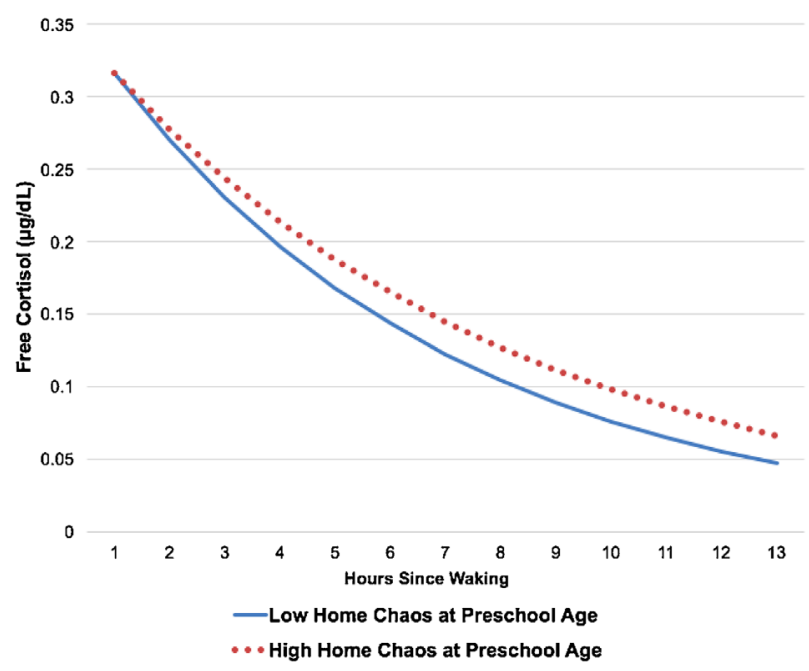

FIGURE 1 Predicted diurnal salivary or free cortisol levels for children in the top and bottom quartiles on household chaos at preschool age
TABLE 5 Cortisol reactivity analysis

\begin{tabular}{|c|c|c|c|c|}
\hline & $\begin{array}{l}\text { Unstandardized } \\
\text { B }\end{array}$ & $\begin{array}{l}\text { Unstandardized } \\
\text { SE }\end{array}$ & $\begin{array}{l}\text { Standardized } \\
\beta\end{array}$ & $T$-score \\
\hline $\begin{array}{l}\text { T2 pubertal } \\
\text { status }\end{array}$ & -79.87 & 51.60 & -0.13 & -1.55 \\
\hline T2 age & 0.77 & 3.71 & 0.02 & 0.21 \\
\hline Female & -71.98 & 57.38 & -0.10 & -1.25 \\
\hline $\begin{array}{l}\text { Non-white } \\
\text { and/or } \\
\text { Hispanic }\end{array}$ & -15.11 & 57.02 & -0.02 & -0.27 \\
\hline $\begin{array}{l}\text { T2 BMI } \\
z \text {-score }\end{array}$ & 26.60 & 31.29 & 0.07 & 0.85 \\
\hline $\begin{array}{l}\text { T2 sleep } \\
\text { disturbance }\end{array}$ & -18.27 & 4.72 & -0.34 & $-3.87^{* * *}$ \\
\hline $\begin{array}{l}\text { T1 income- } \\
\text { to-needs }\end{array}$ & -28.23 & 41.97 & -0.06 & -0.67 \\
\hline $\begin{array}{l}\text { T1 } \\
\text { negative } \\
\text { life } \\
\text { events }\end{array}$ & 11.41 & 5.25 & 0.18 & $2.17^{*}$ \\
\hline $\begin{array}{l}\text { T1 } \\
\text { household } \mathrm{c}\end{array}$ & $\begin{array}{l}17.81 \\
\text { laos }\end{array}$ & 10.74 & 0.14 & 1.66 \\
\hline $\begin{array}{l}\text { T1 family } \\
\text { conflict }\end{array}$ & -85.96 & 47.45 & -0.17 & $-1.81^{\dagger}$ \\
\hline $\begin{array}{r}\text { T2 family } \\
\text { conflict }\end{array}$ & 137.63 & 50.09 & 0.25 & $2.75^{* *}$ \\
\hline
\end{tabular}

Statistical significance indicated by ${ }^{\dagger} p<0.10,{ }^{*} p<0.05,{ }^{* *} p<0.01$, ${ }^{* * *} p<0.001$. T1, preschool assessment; T2, middle childhood asessment.
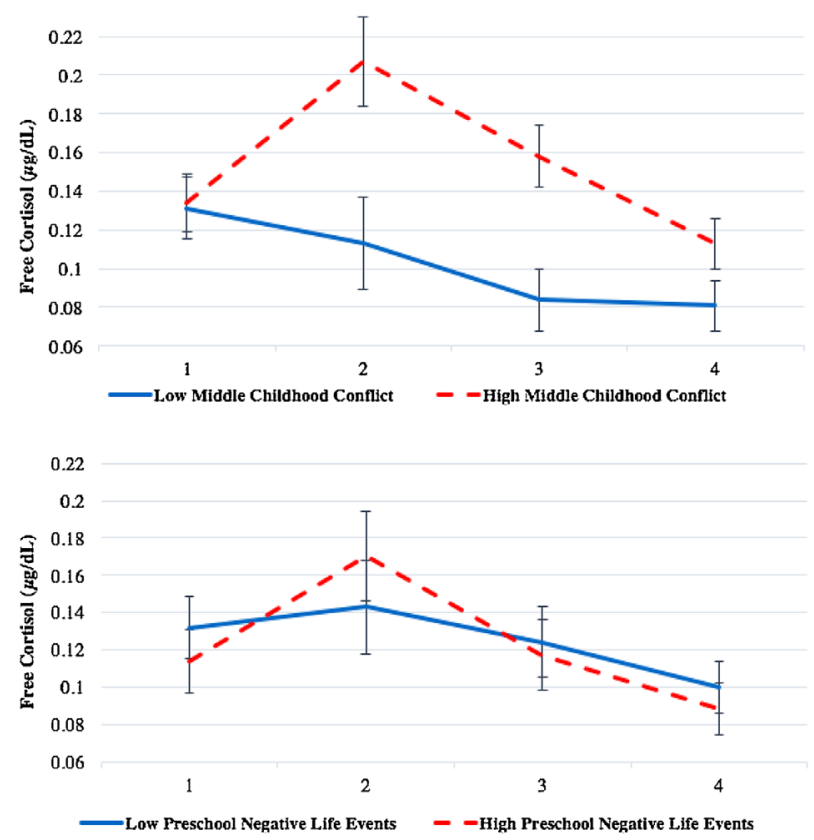

FIGURE 2 Free cortisol levels for children in the top and bottom quartiles of family conflict in middle childhood (top graph) and negative life events in preschool (bottom graph). Cortisol shown for males and females, excluding outliers, adjusted for covariates mentioned in the data analytic plan. Sample 1 was collected immediately preceding the TSST stress task $(0 \mathrm{~min})$, and samples 2 (20-25 min post-baseline), 3 (35-40 min post-baseline), and 4 (55$60 \mathrm{~min}$ post-baseline) were collected after the stressor. Analyses used area under the curve (AUCi) with respect to increase from Sample 1 
Greater T2 family conflict was associated with higher T2 cortisol reactivity, $t(140)=2.74, p<0.01$ (see Table S1). Greater T1 negative life events, $t(140)=2.29, p=0.02$, and greater T1 chaos, $t(140)=2.01$, $p=0.046$, were associated with higher cortisol reactivity. Greater T1 family conflict was associated with lower T2 cortisol reactivity at the level of a trend, $t(140)=-1.95, p=0.053$. Greater T2 sleep disturbance was associated with lower cortisol reactivity, $t(140)=-3.21, p<0.01$.

The overall model significantly predicted cortisol recovery, $F(12$, $138)=21.48, p<0.001$. Greater cortisol reactivity predicted faster cortisol recovery, $t(138)=-14.81, p<0.001$ (see Table S2). No other variable significantly predicted cortisol recovery, $p s>0.10$.

\section{DISCUSSION}

These findings support the hypothesis that the timing and types of psychosocial stressors that are often associated with childhood poverty differentially associate with diurnal cortisol regulation and cortisol reactivity in middle childhood. Identifying chaos and negative life events in preschool and family conflict in middle childhood as regulators of the stress system provides new insight into how specific psychosocial stressors that occur at different points in childhood may impact the biology of the HPA axis. Importantly, the current study examined middle childhood, a time that may be particularly significant for establishing future mental and physical health. Indeed, certain emotional and behavioral problems at this age have been associated with HPA functioning (e.g., Hankin, Badanes, Abela, \& Watamura, 2010; Shirtcliff, Granger, Booth, \& Johnson, 2005). Information on the timing and type of stressors affecting children during this developmental period is essential to develop interventions for children living in low-income households that address environmental changes that may improve physiological regulation and decrease risk for psychopathology and related health problems.

The stressors examined in the current study may differentially influence cortisol production through distinct mechanisms. Negative life events are unique in that they are discrete events that can have an acute negative impact on the child and family but do not necessarily lead to chronic dysfunction in the family. In the current study, more negative life events in preschool predicted higher cortisol reactivity in middle childhood. It could be that the preschool period is a time of sensitization of the HPA axis in response to later stressors. As a result, discrete negative life events in preschool could prime neural circuits and HPA specific elements to respond more strongly to challenges in the future. Potential pathways of neuronal sensitization following early stress include increased dendritic branching in the basolateral amygdala, greater $\mathrm{CRH}$ expression in the central nucleus of the amygdala, and over- or under-recruitment of neural circuitry underlying HPA neuroendocrine stress responses (Ulrich-Lai \& Herman, 2009). Unlike chronic stressors, negative life events due to their episodic nature could lead to augmentation of the HPA neuroendocrine stress response rather than a diminution, as observed with chronic repetitive stress exposure. Interestingly, our follow-up analyses indicated that negative life events were more predictive of cortisol reactivity than recovery, suggesting different factors affect cortisol reactivity and recovery in unique ways. A possible pathway by which negative life events during preschool may lead to heightened cortisol reactivity is through parenting. The stress of these negative life events could impact caregiver stress levels and parenting quality over time (Essex, Klein, Cho, \& Kalin, 2002; Fisher \& Stoolmiller, 2008), which might lead to disruptions in cortisol reactivity in middle childhood.

Chaos may have a specific impact on diurnal cortisol levels as chaos is less acutely threatening to the child and the stability of the family compared to negative life events and family conflict. Rather, chaos may be viewed as a chronic condition of unpredictability and uncontrollability, producing many mild stressors throughout the day and disrupting routines that would otherwise facilitate greater stability in stress biology. Chaos and unpredictable schedules likely contribute to blunted diurnal patterns observed in certain pediatric populations, especially if there is great variability in sleep schedules (Gunnar \& Vazquez, 2001). The current analyses suggested that the chaos may not be related to morning cortisol levels, but rather related to the blunting of the cortisol across the day. The frequent, mild stress of chaos may lead to higher cortisol levels during the day that prevent cortisol from reaching its typical nadir in the evening. Upregulation of cortisol production across the day may be helpful to deal with stressors in the short-term, but could be deleterious over time (internalizing disorders, growth and development; Tarullo \& Gunnar, 2006). Since chaos is likely a milder stressor than family conflict, especially in regard to social threat, it may take more time for potential associations between chaos and observed physiology. As with negative life events, chaos could impact child cortisol levels over time through alterations in caregiver stress and emotional availability, parenting quality, and the caregiver's attentiveness to the child's cues. Consistent with previous early life stress reports (Gunnar \& Vazquez, 2006; Tarullo \& Gunnar, 2006), chaos during preschool may be more associated with diurnal cortisol production. There is also evidence from this study that greater chaos in preschool is associated with greater cortisol reactivity but not recovery in middle childhood, which could serve to heighten responsivity to respond to stressors to prepare the child for a future chaotic environment in middle childhood.

A possible mechanism by which current family conflict could influence cortisol reactivity is through the child consciously or unconsciously interpreting family conflict as a stressor that is threatening to both the child and family's safety and stability. As a result, family conflict in the home might make children hyper-vigilant to social threats, interpret those threats more negatively, have a stronger emotional reaction, and show a profile of heightened autonomic and neuroendocrine HPA response in the face of future social threats (Luecken et al., 2013; Miller et al., 2011). More research needs to be done to understand whether conscious or unconscious interpretations of stress in children are associated with cortisol functioning, but this is a possible mechanism by which family stress affects the HPA axis. The current findings suggested that current family conflict is a better predictor of cortisol reactivity than preschool family conflict. It could be that threat detection and stress-mediating systems may dynamically adapt to relationship threats such that 
current conflict is a better predictor of functioning than past conflict or change in conflict. Our results were similar to a study that reported that increased distress responses to interparental conflict were associated with heightened cortisol reactivity to a simulated parent conflict task (Davies, Sturge-Apple, Cicchetti, \& Cummings, 2008), suggesting that heightened emotional responsiveness governed by limbic brain structures could be a pathway by which increased family conflict could lead to simultaneous increases in cortisol reactivity. Another possibility is that current family conflict leads to the perception of less reliable social support, either consciously or unconsciously, reinforcing the need to upregulate cortisol responsiveness in order to cope with stressors alone (Tarullo \& Gunnar, 2006). During middle childhood, this may become a particularly salient issue as children begin to develop their own peer social networks outside the family system. In our study, the social component of the TSST-C may have been more relevant for eliciting greater cortisol responses in children exposed to family conflict than exposed to stressors that are less socially threatening. Family conflict may have unique associations with social functioning compared to the other stressors assessed, which could have implications for stress biology in social situations. Current family conflict was also associated with reactivity and not recovery, suggesting different mechanisms for affecting these components of HPA responses to stress. There was a trend-level finding that greater family conflict in preschool was related to a more blunted cortisol response to the TSST, which could indicate that earlier family conflict is related to blunting of cortisol reactivity while current conflict is associated with heightened reactivity. However, this result must be replicated in future work.

The findings of this study inform important questions in stress biology and developmental psychopathology by identifying sensitive periods for the shaping of HPA axis regulation at different points in development. The specificity of both psychosocial stressor type and the developmental timing of such stressors in relation to cortisol reactivity versus diurnal cortisol generates additional important questions concerning critical HPA axis regulators and sensitive periods of development. First, are these findings specific to early-middle childhood, or would we observe similar associations in adolescence or adulthood? It could be that adolescents and adults who were exposed to psychosocial stressors early in life exhibit cortisol profiles similar to younger children with adverse experiences. Alternatively, profiles could appear different given that developmental processes such as pubertal maturation may reshape biological systems to adapt to the current environment, which may have changed since early childhood, and also to a changing social environment (Doom, Hostinar, VanZomeren-Dohm, \& Gunnar, 2015). For example, our results differ from those of Bosch et al. (2012) which report no association between stressors from ages 0-5 years and cortisol reactivity in adolescence. However, the timing of assessment in adolescence may be an explanation for this difference, as greater stressors from ages 6-11 were associated with increased cortisol reactivity in adolescence (Bosch et al., 2012). Similarly, our findings of increased cortisol reactivity for children from families with greater family conflict differed from others in the literature (Davies et al., 2007, Saxbe et al.,
2012), but this may also be due to the timing of the assessment (middle childhood vs. early childhood or adolescence) and the nature of the stress task (social evaluation by an unfamiliar adult vs. family conflict task).

Second, what aspects of these stressors make them critical regulators of current and future HPA functioning? Current family conflict may be consciously or unconsciously interpreted as a threat to the family and the self, leading to increased vigilance, difficulty forming stress-buffering social relationships, and greater stress reactivity (Miller et al., 2011). Negative life events and chaos during the preschool years may signal that the environment is unpredictable and unsafe, which both limits young children's options for obtaining help in managing their stress (Gunnar, 1998) and also programs physiological systems to prepare for a similar environment in the future. However, negative events and chaos may be differentially associated with HPA functioning due to the differences in chronicity of these stressors. For example, negative life events may be more acute and severe stressors, while chaos may be a more smoldering, chronic, but less severe psychosocial stressor.

It will be important for interventions, particularly those focused on alleviating the effects of stress on children growing up in poverty, to target these critical regulators during sensitive periods to optimize effectiveness. The findings of this study certainly support the importance of preschool as a sensitive period and suggest that chaos and negative life events are psychosocial stressors that regulate development of the HPA axis longitudinally. Finally, how can interventions address the specificity of psychosocial stressors? There is evidence suggesting that basal cortisol and cortisol reactivity can be differentially affected by stress and psychopathology (e.g., Alink et al., 2008; Tomiyama et al., 2012), and it will be important to consider how interventions can address the effects of different stressors on cortisol activity. Of course, different aspects of HPA activity serve different physiological purposes, and further research is needed to understand why and how stressors may uniquely affect basal cortisol vs. cortisol reactivity and how interventions may address these differences.

Finally, it is important to note that although scientists often discuss differences between groups as normative regulation versus "dysregulation," such differences may also represent physiological changes made to adapt to different types of environments that vary in the severity of stress. For example, high stress reactivity has been linked to maladaptive outcomes in high adversity contexts and enhanced adaptation in low adversity contexts (Obradović, Bush, Stamperdahl, Adler, \& Boyce, 2010). Thus, it may be more appropriate to think about biological sensitivity to context and conditional adaptations to current and future stressors (Boyce \& Ellis, 2005; Obradović et al., 2010). In this study, high levels of family conflict are concurrently associated with increased reactivity to a psychosocial stressor. In the case of family conflict, neural circuitry processing cues of threat and negative emotion may be primed by recent events in the household to detect and respond to cues that may signal a need for an individual to be prepared to protect themselves from an immediate threat within the family (Pollak, 2008), which is a place the child might usually expect to be safe. Additionally, children who experience greater chaos and 
negative life events in preschool may detect an unpredictable and threatening immediate and external environment early in life. Their developing stress-mediating systems may program cortisol reactivity and diurnal cortisol regulation in a way that will help them adapt to similar chaotic environments or negative life events in the future. In fact, it may be that these predictive adaptations made to survive in stressful environments are maladaptive and lead to mental and physical health problems when the current or future environment does not match the previous adaptations (Doom \& Gunnar, 2013). Alternatively, these adaptations may be intended for short-term survival as it may be metabolically costly to have enhanced responsiveness or higher evening cortisol levels for long periods (McEwen, 1998). Therefore, if the stressful environment and the concurrent physiological adaptations persist beyond a short period of time, these adaptations may ultimately become maladaptive for mental and physical health (McEwen, 1998). It is also essential to remember that measuring cortisol levels assesses just one aspect of the body's stress-mediating systems. When thinking about upregulation or downregulation of physiological systems, we must consider higher levels of the HPA axis, such as the hippocampus, the hypothalamus and the pituitary, and inputs from systems ranging from the immune system to the sympathetic nervous system to emotion and fear circuitry in the brain. Changes in functioning in one system will likely impact functioning in a number of stress-mediating systems across development (Doom \& Gunnar, 2013; Joëls \& Baram, 2009).

This study had limitations that must be discussed when interpreting the results. First, the income-to-needs ratio was used as a proxy for the income component of SES as we did not have more detailed measures of financial strain at both time points. Although there is not a consensus on the best way to measure the income component of SES, we have attempted to use an objective measure incorporating income and family size in order to control for effects that may be due solely to low resources rather than poverty-related stressors specifically. It is important to note that this study did not have any primary caregivers who attained a 4-year college degree, and nearly all families were from low-income backgrounds, so as planned, there was not great variability within our low-income sample. Future studies should utilize measures that assess aspects of living in poverty that are not captured by either negative life events measures or the income-to-needs ratio, such as perceived economic insufficiency, which has been associated with child cortisol levels (Blair et al., 2011). Second, there are clearly many aspects of the environment that could contribute to differences in HPA reactivity and regulation besides those measured here. For example, Blair et al. (2011) reported that the number of adult exits from the home was associated with child cortisol levels. Although some of the specific stressors may be reflected in the constructs we assessed, we did not measure all of these potential stressors specifically. Other stressors should be measured at a more granular level in future studies of children from low-income backgrounds. Third, the stressor task was a test of reactivity to psychosocial stress, which may constrain interpretation to events that involve negative emotion and fear of social evaluation as opposed to a physical stressor (Dickerson \& Kemeny, 2004). However, as social evaluation is salient to children in social groups and school contexts, this is likely a useful context to understand stress reactivity. Fourth, the report of psychosocial stress exposures is from parents and not children, whose interpretation is likely critical in order to understand how the stressors are interpreted and how the system adapts to challenges. However, as these children were too young to report in preschool and still fairly young in middle childhood, the parent may have provided the most accurate information about the environment. Fifth, the timing of the diurnal cortisol sampling and potential compliance issues could have affected our results. For example, more chaotic families may have had more difficulty with compliance, which could have affected their diurnal cortisol curves, although concurrent family chaos did not predict diurnal cortisol slope in this sample. In addition, we asked families to collect cortisol during the first $30 \mathrm{~min}$, which could introduce variability in cortisol levels during that time due to the cortisol awakening response. We attempted to limit this possible effect by estimating $60 \mathrm{~min}$ post-awakening as our morning cortisol intercept, but this variability could impact our results. Finally, additional longitudinal assessments on similar cohorts are needed to understand these associations over time at different points in development. However, we hope that these analyses prompt future longitudinal work on specific types and timing of stressors that may differentially affect HPA responses.

These results are especially important for informing interventions for children experiencing poverty and related stressors. A recent systematic review of psychosocial intervention studies in children stated that 18 out of 19 papers reported differences in cortisol levels between the intervention and control groups, although the types of changes were inconsistent (Slopen, McLaughlin, \& Shonkoff, 2014). However, all eight of the studies that included a low-risk comparison group found evidence that the intervention group was similar to the low-risk group and differed from the high-risk control group. Thus, there may be considerable plasticity even after experiencing stressors during sensitive periods. Future empirical studies are needed to follow up the current cohort and other cohorts from low-income backgrounds to understand whether these HPA alterations that are associated with preschool and middle childhood stressors persist and whether they are associated with future mental and physical health problems. Future research must also measure a wider variety of environmental and social challenges experienced by children living in poverty in order to understand the long-term effects of these critical regulators of the HPA axis.

\section{8 | CONCLUSIONS}

Our findings in low-income school-aged children who participated in Head Start suggest that both developmental timing and type of psychosocial stressors experienced may associate with different aspects of HPA regulation. Specifically, more chaos in the home during preschool predicted a more blunted diurnal cortisol slope in middle childhood. In addition, greater negative life events during preschool and more concurrent family conflict were associated with an increased cortisol response to psychosocial stress in middle childhood. These findings will be 
important for informing interventions that seek to promote adaptive physiological regulation in children from low-income backgrounds.

\section{ACKNOWLEDGMENTS}

This research was supported by funding from NICHD/NIDDK (R01DK095695 [PI: Miller and Lumeng], R21DK090718 [PI: Miller and Lumeng], RC1DK086376 [PI: Lumeng], and F32HD088029 [PI: Doom]), and the American Heart Association (10GRNT4460043 [PI: Miller]).

\section{ORCID}

Jenalee R. Doom iD http://orcid.org/0000-0003-2857-0817

\section{REFERENCES}

Alink, L. R., van IJzendoorn, M. H., Bakermans-Kranenburg, M. J., Mesman, J., Juffer, F., \& Koot, H. M. (2008). Cortisol and externalizing behavior in children and adolescents: Mixed meta-analytic evidence for the inverse relation of basal cortisol and cortisol reactivity with externalizing behavior. Developmental Psychobiology, 50(5), 427-450.

Beavers, W., Hampson, R., \& Hulgus, Y. (1991). Beavers systems model: Observational and self-report scales. Dallas, TX: Southwest Family Institute.

Blair, C., Berry, D., Mills-Koonce, R., \& Granger, D. (2013). Cumulative effects of early poverty on cortisol in young children: Moderation by autonomic nervous system activity. Psychoneuroendocrinology, 38(11), 2666-2675. https://doi.org/10.1016/j.psyneuen.2013.06.025

Blair, C., Granger, D., \& Razza, R. P. (2005). Cortisol reactivity is positively related to executive function in preschool children attending head start. Child Development, 76(3), 554-567.

Blair, C., Raver, C., Granger, D., Mills-Koonce, R., Hibel, L., \& the Family Life Project Key, I. (2011). Allostasis and allostatic load in the context of poverty in early childhood. Development and Psychopathology, 23(3), 845-857. https://doi.org/10.1017/S0954579411000344

Bosch, N. M., Riese, H., Reijneveld, S. A., Bakker, M. P., Verhulst, F. C., Ormel, J., \& Oldehinkel, A. J. (2012). Timing matters: Long term effects of adversities from prenatal period up to adolescence on adolescents' cortisol stress response. The TRAILS study. Psychoneuroendocrinology, 37(9), 1439-1447. https://doi.org/10.1016/j.psyneuen.2012.01.013

Boyce, W. T., \& Ellis, B. J. (2005). Biological sensitivity to context: I. An evolutionary-developmental theory of the origins and functions of stress reactivity. Development and Psychopathology, 17(02), 271-301.

Buske-Kirschbaum, A., Jobst, S., Wustmans, A., Kirschbaum, C., Rauh, W., \& Hellhammer, D. (1997). Attenuated free cortisol response to psychosocial stress in children with atopic dermatitis. Psychosomatic Medicine, 59(4), 419-426. https://doi.org/10.1097/00006842-199707000-00012

Chen, E., Cohen, S., \& Miller, G. E. (2010). How low socioeconomic status affects 2-year hormonal trajectories in children. Psychological Science, 21(1), 31-37. https://doi.org/10.1177/0956797609355566

Davies, P. T., Sturge-Apple, M. L., Cicchetti, D., \& Cummings, E. M. (2007). The role of child adrenocortical functioning in pathways between interparental conflict and child maladjustment. Developmental Psychology, 43(4), 918-930. https://doi.org/10.1037/0012-1649. 43.4.918

Davies, P. T., Sturge-Apple, M. L., Cicchetti, D., \& Cummings, E. M. (2008). Adrenocortical underpinnings of children's psychological reactivity to interparental conflict. Child Development, 79(6), 1693-1706. https:// doi.org/10.1111/j.1467-8624.2008.01219.x
Dickerson, S. S., \& Kemeny, M. E. (2004). Acute stressors and cortisol responses: A theoretical integration and synthesis of laboratory research. Psychological Bulletin, 130(3), 355-391. https://doi.org/ 10.1037/0033-2909.130.3.355

Doom, J. R., Cicchetti, D., Rogosch, F. A., \& Dackis, M. N. (2013). Child maltreatment and gender interactions as predictors of differential neuroendocrine profiles. Psychoneuroendocrinology, 38(8), 1442-1454. http://doi.org/10.1016/j.psyneuen.2012.12.019

Doom, J. R., \& Gunnar, M. R. (2013). Stress physiology and developmental psychopathology: Past, present and future. Development and Psychopathology, 25(402), https://doi.org/10.1017/s0954579413000667

Doom, J. R., Hostinar, C. E., VanZomeren-Dohm, A. A., \& Gunnar, M. R. (2015). The roles of puberty and age in explaining the diminished effectiveness of parental buffering of HPA reactivity and recovery in adolescence. Psychoneuroendocrinology, 59, 102-111. https://doi.org/ 10.1016/j.psyneuen.2015.04.024

Essex, M. J., Klein, M. H., Cho, E., \& Kalin, N. H. (2002). Maternal stress beginning in infancy may sensitize children to later stress exposure: Effects on cortisol and behavior. Biological Psychiatry, 52(8), 776-784.

Evans, G. W. (2004). The environment of childhood poverty. American Psychologist, 59(2), 77-92. https://doi.org/10.1037/0003-066X.59.2.77

Evans, G. W., \& Kim, P. (2007). Childhood poverty and health: Cumulative risk exposure and stress dysregulation. Psychological Science, 18(11), 953-957. https://doi.org/10.1111/j.1467-9280.2007.02008.x

Evans, G. W., Li, D., \& Whipple, S. S. (2013). Cumulative risk and child development. Psychological Bulletin, 139(6), 1342.

Feng, X., Wang, L., Yang, S., Qin, D., Wang, J., Li, C., \& Hu, X. (2011). Maternal separation produces lasting changes in cortisol and behavior in rhesus monkeys. Proceedings of the National Academy of Sciences, 108(34), 14312-14317.

Fernald, L., \& Gunnar, M. R. (2009). Effects of a poverty-alleviation intervention on salivary cortisol in very low-income children. Social Science \& Medicine (1982), 68(12), 2180-2189. https://doi.org/ 10.1016/j.socscimed.2009.03.032

Fisher, P. A., \& Stoolmiller, M. (2008). Intervention effects on foster parent stress: Associations with children's cortisol levels. Development and Psychopathology, 20(3), 1003-1021. https://doi.org/10.1017/S0954579408000473

Fries, E., Hesse, J., Hellhammer, J., \& Hellhammer, D. H. (2005). A new view on hypocortisolism. Psychoneuroendocrinology, 30(10), 1010-1016.

Granger, D. A., Hibel, L. C., Fortunato, C. K., \& Kapelewski, C. H. (2009). Medication effects on salivary cortisol: Tactics and strategy to minimize impact in behavioral and developmental science. Psychoneuroendocrinology, 34(10), 1437-1448.

Gunnar, M., \& Quevedo, K. (2007). The neurobiology of stress and development. Annual Review of Psychology, 58, 145-173. https://doi. org/10.1146/annurev.psych.58.110405.085605

Gunnar, M. R. (1998). Quality of early care and buffering of neuroendocrine stress reactions: Potential effects on the developing human brain. Preventive Medicine, 27(2), 208-211.

Gunnar, M. R., Doom, J. R., \& Esposito, E. A. (2015). Psychoneuroendocrinology of stress. Handbook of child psychology and developmental science. Hoboken, NJ: Wiley.

Gunnar, M. R., \& Vazquez, D. (2006). Stress neurobiology and developmental psychopathology. In D. Cicchetti, \& D. Cohen (Eds.), Developmental psychopathology (Vol. 2). Hoboken, NJ: Wiley.

Gunnar, M. R., \& Vazquez, D. M. (2001). Low cortisol and a flattening of expected daytime rhythm: Potential indices of risk in human development. Development and Psychopathology, 13(03), 515-538.

Gunnar, M. R., \& White, B. P. (2001). Salivary cortisol measures in infant and child assessment. In L. T. S. P. S. Zeskind (Ed.), Biobehavioral assessment of the infant (pp. 167-189). New York, NY, US: Guilford Press.

Hampson, R. B., Hulgus, Y. F., \& Beavers, W. R. (1991). Comparisons of selfreport measures of the beavers systems model and olson's circumplex model. Journal of Family Psychology, 4(3), 326. 
Hankin, B. L., Badanes, L. S., Abela, J. R. Z., \& Watamura, S. E. (2010) Hypothalamic-pituitary-adrenal axis dysregulation in dysphoric children and adolescents: Cortisol reactivity to psychosocial stress from preschool through middle adolescence. Biological Psychiatry, 68(5), 484-490.

Hruschka, D. J., Kohrt, B. A., \& Worthman, C. M. (2005). Estimating between- and within-individual variation in cortisol levels using multilevel models. Psychoneuroendocrinology, 30(7), 698-714. https:// doi.org/10.1016/j.psyneuen.2005.03.002

Jenness, J. L., \& McLaughlin, K. A. (2015). Towards a person-centered approach to the developmental psychopathology of trauma. Social Psychiatry and Psychiatric Epidemiology, 50(8), 1219-1221. https://doi. org/10.1007/s00127-015-1095-1

Joëls, M., \& Baram, T. Z. (2009). The neuro-symphony of stress. Nature Reviews Neuroscience, 10(6), 459-466. https://doi.org/10.1038/ $\mathrm{nrn} 2632$

Kilmer, R. P., Cowen, E. L., Wyman, P. A., Work, W. C., \& Magnus, K. B. (1998). Differences in stressors experienced by urban African American, White, and Hispanic children. Journal of Community Psychology, 26(5), 415-428. https://doi.org/10.1002/(SICI)1520-6629(199809) 26:5<415::AID-JCOP2>3.0.CO;2-

Kuczmarski, R. J., Ogden, C. L., Guo, S. S., Grummer-Strawn, L. M., Flegal, K. M., Mei, Z., .. . Johnson, C. L. (2002). 2000 CDC growth charts for the United States: Methods and development. Vital and Health Statistics. Series 11, Data From the National Health Survey, 246, 1-190.

Kumari, M., Badrick, E., Ferrie, J., Perski, A., Marmot, M., \& Chandola, T. (2009). Self-reported sleep duration and sleep disturbance are independently associated with cortisol secretion in the Whitehall II study. The Journal of Clinical Endocrinology \& Metabolism, 94(12), 4801-4809.

Lopez-Duran, N. L., Hajal, N. J., Olson, S. L., Felt, B. T., \& Vazquez, D. M. (2009). Individual differences in cortisol responses to fear and frustration during middle childhood. Journal of Experimental Child Psychology, 103(3), 285-295. https://doi.org/10.1016/j.jecp.2009.03.008

Luecken, L. J., Roubinov, D. S., \& Tanaka, R. (2013). Childhood family environment, social competence, and health across the lifespan. Journal of Social and Personal Relationships, 30(2), 171-178.

Lumeng, J. C., Miller, A., Peterson, K. E., Kaciroti, N., Sturza, J., Rosenblum, K., \& Vazquez, D. M. (2014). Diurnal cortisol pattern, eating behaviors and overweight in low-income preschool-aged children. Appetite, 73, 65-72. https://doi.org/10.1016/j.appet.2013.10.016

Lupien, S. J., King, S., Meaney, M. J., \& McEwen, B. S. (2001). Can poverty get under your skin? Basal cortisol levels and cognitive function in children from low and high socioeconomic status. Development and Psychopathology, 13(3), 653-676. https://doi.org/10.1017/S0954579401003133

Lupien, S. J., McEwen, B. S., Gunnar, M. R., \& Heim, C. (2009). Effects of stress throughout the lifespan on the brain, behaviour and cognition. Nature Reviews Neuroscience, 10(6), 434-445.

Massey, A. J., Campbell, B. K., Raine-Fenning, N., Pincott-Allen, C., Perry, J., \& Vedhara, K. (2016). Relationship between hair and salivary cortisol and pregnancy in women undergoing IVF. Psychoneuroendocrinology, 74, 397-405.

Matheny, A. P., Wachs, T. D., Ludwig, J. L., \& Phillips, K. (1995). Bringing order out of chaos: Psychometric characteristics of the confusion, hubbub, and order scale. Journal of Applied Developmental Psychology, 16(3), 429-444. https://doi.org/10.1016/0193-3973(95) 90028-4

McEwen, B. S. (1998). Stress, adaptation, and disease: Allostasis and allostatic load. Annals of the New York Academy of Sciences, 840(1), 33-44. https://doi.org/10.1111/j.1749-6632.1998.tb09546.x

McEwen, B. S., \& Stellar, E. (1993). Stress and the individual: Mechanisms leading to disease. Archives of Internal Medicine, 153(18), 2093-2101. https://doi.org/10.1001/archinte.153.18.2093

Miller, A. L., Song, J. H., Sturza, J., Lumeng, J. C., Rosenblum, K., Kaciroti, N., \& Vazquez, D. M. (2017). Child cortisol moderates the association between family routines and emotion regulation in low-income children. Developmental Psychobiology, 59, 99-110. https://doi.org/ 10.1002/dev.21471

Miller, G. E., Chen, E., \& Parker, K. J. (2011). Psychological stress in childhood and susceptibility to the chronic diseases of aging: Moving towards a model of behavioral and biological mechanisms. Psychological Bulletin, 137(6), 959-997. https://doi.org/10.1037/ a0024768

Miller, G. E., Chen, E., \& Zhou, E. S. (2007). If it goes up, must it come down? Chronic stress and the hypothalamic-pituitary-adrenocortical axis in humans. Psychological Bulletin, 133(1), 25-45.

Morris, N. M., \& Udry, J. R. (1980). Validation of a self-administered instrument to assess stage of adolescent development. Journal of Youth and Adolescence, 9(3), 271-280.

Obradović, J., Bush, N. R., Stamperdahl, J., Adler, N. E., \& Boyce, W. T. (2010). Biological sensitivity to context: The interactive effects of stress reactivity and family adversity on socioemotional behavior and school readiness. Child Development, 81(1), 270-289.

Owens, J. A., Spirito, A., \& McGuinn, M. (2000). The Children's Sleep Habits Questionnaire (CSHQ): Psychometric properties of a survey instrument for school-aged children. Sleep, 23(8), 1043-1051.

Pendry, P., \& Adam, E. K. (2007). Associations between parents' marital functioning, maternal parenting quality, maternal emotion and child cortisol levels. International Journal of Behavioral Development, 31(3), 218-231.

Pollak, S. D. (2008). Mechanisms linking early experience and the emergence of emotions: Illustrations from the study of maltreated children. Current Directions in Psychological Science, 17(6), 370-375. https://doi.org/10.1111/j.1467-8721.2008.00608.x

Pruessner, J. C., Kirschbaum, C., Meinlschmid, G., \& Hellhammer, D. H. (2003). Two formulas for computation of the area under the curve represent measures of total hormone concentration versus time-dependent change. Psychoneuroendocrinology, 28(7), 916-931.

Repetti, R. L., Taylor, S. E., \& Seeman, T. E. (2002). Risky families: Family social environments and the mental and physical health of offspring. Psychological Bulletin, 128(2), 330-366. https://doi.org/10.1037/ 0033-2909.128.2.330

Saxbe, D. E., Margolin, G., Spies Shapiro, L. A., \& Baucom, B. R. (2012). Does dampened physiological reactivity protect youth in aggressive family environments? Child Development, 83(3), 821-830. https://doi.org/ 10.1111/j.1467-8624.2012.01752.x

Shirtcliff, E. A., Granger, D. A., Booth, A., \& Johnson, D. (2005). Low salivary cortisol levels and externalizing behavior problems in youth. Development and Psychopathology, 17(01), 167-184.

Simmons, J. G., Badcock, P. B., Whittle, S. L., Byrne, M. L., Mundy, L., Patton, G. C., . . A Allen, N. B. (2016). The lifetime experience of traumatic events is associated with hair cortisol concentrations in community-based children. Psychoneuroendocrinology, 63, 276-281. https://doi.org/ 10.1016/j.psyneuen.2015.10.004

Slatcher, R. B., \& Robles, T. F. (2012). Preschoolers' everyday conflict at home and diurnal cortisol patterns. Health Psychology, 31(6), 834

Slopen, N., McLaughlin, K. A., \& Shonkoff, J. P. (2014). Interventions to improve cortisol regulation in children: A systematic review. Pediatrics, 133(2), 312-326. https://doi.org/10.1542/peds.20131632

Sturge-Apple, M. L., Davies, P. T., Cicchetti, D., Hentges, R. F., \& Coe, J. L. (2017). Family instability and children's effortful control in the context of poverty: Sometimes a bird in the hand is worth two in the bush. Development and Psychopathology, 29, 685-696.

Tarullo, A. R., \& Gunnar, M. R. (2006). Child maltreatment and the developing HPA axis. Hormones and Behavior, 50(4), 632-639.

Tomiyama, A. J., O’Donovan, A., Lin, J., Puterman, E., Lazaro, A., Chan, J., . . . Epel, E. (2012). Does cellular aging relate to patterns of allostasis? An examination of basal and stress reactive HPA axis activity and telomere 
length. Physiology \& Behavior, 106(1), 40-45. https://doi.org/10.1016/j. physbeh.2011.11.016

Ulrich-Lai, Y. M., \& Herman, J. P. (2009). Neural regulation of endocrine and autonomic stress responses. Nature Reviews Neuroscience, 10(6), 397-409. https://doi.org/10.1038/nrn2647

Vliegenthart, J., Noppe, G., van Rossum, E. F. C., Koper, J. W., Raat, H., \& van den Akker, E. L. T. (2016). Socioeconomic status in children is associated with hair cortisol levels as a biological measure of chronic stress. Psychoneuroendocrinology, 65, 9-14. https://doi.org/10.1016/j.psyneuen.2015.11.022

The Wechsler Individual Achievement Test-Third Edition (WIAT III). (2009). San Antonio: Pearson.

Work, W. C., Cowen, E. L., Parker, G. R., \& Wyman, P. A. (1990). Stress resilient children in an urban setting. Journal of Primary Prevention, 11(1), 3-17. https://doi.org/10.1007/BF01324858

\section{SUPPORTING INFORMATION}

Additional Supporting Information may be found online in the supporting information tab for this article.

How to cite this article: Doom JR, Cook SH, Sturza J, et al. Family conflict, chaos, and negative life events predict cortisol activity in low-income children. Developmental Psychobiology. 2018;60:364-379. https://doi.org/10.1002/dev.21602 